\title{
Nuovi modelli di business nel capitalismo globale della conoscenza in rete \\ Enzo Rullani \\ Nuovi modelli di business nel capitalismo globale della conoscenza in rete
}

\author{
Enzo Rullani
}

Un vero viaggio di scoperta
non è visitare nuovi luoghi
ma avere nuovi occhi
Marcel Proust

\begin{abstract}
Dal 2000 in poi le imprese sono impegnante in una transizione verso il paradigma emergente (il capitalismo globale della conoscenza in rete) che somma in modo inestricabile le due dimensioni del "digitale" e del "globale". Rompendo con le preesistenze e segnando discontinuità importanti sia nellesperienza degli imprenditori e delle organizzazioni produttive, sia in quella degli altri attori della società attuale: i manager, i lavoratori dipendenti, i risparmiatori, i fornitori e gli attori del circuito distributivo, i consumatori, le istituzioni locali e nazionali.

Queste discontinuità nascono soprattutto dalle trasformazioni che la transizione digitale/globale esercita sulla conoscenza, per cui tutta una serie di conoscenze (quelle traducibili in un codice astratto) diventano mobili, potendo essere replicate e trasferite a costo zero, in tempo reale, da un punto allaltro del pianeta, ovunque esistano le capacità per utilizzarle. Un'ingente massa di conoscenze pratiche - che finora erano rimaste ancorate alle persone e ai contesti di origine - possono cosi spostarsi ed essere attratte dai paesi e dai luoghi in cui il loro uso è più conveniente. Altre conoscenze (generative) restano invece ancorate alle persone e ai contesti che le hanno prodotte, dando luogo a vantaggi distintivi importanti nelle nuove value chains globali.

Per portare avanti una strategia pro-attiva che vada oltre le pre-esistenze e sperimenti nuovi modelli di business, bisogna non solo innovare, ma scegliere quelle innovazioni che sono adatte a sfruttare l'energia delle cinque grandi onde della transizione in corso (networking, automazione, globalizzazione, worldmaking, ripersonalizzazione).

$\grave{E}$ tutto un mondo che sta cambiando, facendo emergere anche nuovi problemi, alcuni dei quali vanno oltre le capacità di intervento della singola imprese e richiedono di essere trattati da soggetti collettivi con cui le impresa possono responsabilmente collaborare, esplorando nuove possibilità anche in termini di business. Bisogna infatti imparare a rilevare per tempo, e trovare rimedi adeguati, alla svalorizzazione progressiva del lavoro esecutivo; alla crescita del rischio diffuso (che può bloccare gli investimenti); allemergere di forme nuove di insostenibilità (controllo monopolistico sui dati e sulla privacy, influenze poco trasparenti nelle relazioni in rete); e alla crescita delle disuguaglianze sociali, che nascono dal procedere selettivo - non inclusivo delle innovazioni digitali/globali. I nuovi modelli di business hanno qualche chance di successo se fin dall'inizio di misurano con questi problemi e con le loro soluzioni possibili.
\end{abstract}


Sinergie

Vol. 37, Issue 1, 2019

Since 2000, firms have been facing a transition that is gradually weakening the entrepreneurial culture and the competitive assets inherited from two historical paradigms of the nineteenth century: the Fordism of big auto-referential organizations (1900-1970) and the flexible capitalism of industrial districts and lean production (1970-2000). The converging processes of market globalization and the digitalization of knowledge have long highlighted the existence of a new paradigm: the global capitalism of networked knowledge. Even though this paradigm is still incomplete and contradictory, it is sufficient to destabilize the status quo, urging firms to search for new balances and new relationship systems.

Because of this transition, business models are changing in all industries of all dimensions. Due to the implosion of distances, global supply chains are adopting an increasingly intense division of labor by focusing on territories able to attract specific phases and functions of the supply chain activities. At the same time, the enlargement of markets, the replication at no cost of apps, data and digital programs, and the speeding up of responses according to on-demand production are enhancing the importance of market multipliers, which make it possible to use the same knowledge and the same standards in large volumes.

In the new digital/global paradigm, disruptive innovations are destined to spread rapidly and exponentially, feeding a "boom" that prepares a subsequent downfall. Giving rise to a messy swarm of social and personal discomforts, associated with processes of exclusion and technological unemployment. Putting together the winners and the losers of this transition is not only a political challenge, but also a managerial and social challenge since it is necessary to search for innovations that are not only relevant, but also sustainable from different perspectives.

The question is: which companies and countries can gain an advantage by actively participating, before and better than others, to this evolution? In particular, what can Italian firms do to increase their ability to generate value and enhance the attractiveness of our production system, by proposing business models appropriate for the digital/global environment that is emerging in the present and will greatly affect the near future?

Key words: digitale; transizione; modelli dibusiness; networking; ri-personalizzazione; lavoro

\section{Avere nuovi occhi: $i$ tre diversi modi con cui la digitalizzazione crea valore}

Viviamo in un'epoca di grandi cambiamenti, in cui il presente, carico delle sue contraddizioni, si confronta con il futuro possibile lungo una linea di faglia che segna insieme progressi e rotture, spesso intrecciati in modo inestricabile. In ogni discussione, tutti sentono, infatti, il bisogno di fare riferimento a quello che eravamo, in passato, e a quello che possiamo o dobbiamo diventare in futuro. Invece, dobbiamo imparare a guardare il presente con "nuovi occhi", uscendo dalla narrazione finora prevalente (quella della crisi) per adottare una visione diversa: quella della transizione che ci allontana dai vecchi paradigmi del secolo scorso (il fordismo del periodo 1900-1970, e il capitalismo distrettuale degli anni 1970-2000) per portarci a costruire un nuovo paradigma: il capitalismo globale della 
conoscenza in rete, che ha iniziato ad emergere dal 2000 in poi ed è adesso il porto di arrivo della transizione digitale/globale in corso.

Il salto evolutivo imposto dalla transizione digitale/globale ha un grande rilievo per le politiche economiche e industriali da portare avanti. L'Italia, con le sue specificità (eco-sistemi locali, impresa diffusa, piccola dimensione, "capitalismo senza capitali" ecc.) deve infatti imparare a gestire la propria unicità, cercando di andare avanti lungo una strada che sarà necessariamente diversa da quella degli altri. Anche se dall'esperienza altrui si può e si deve imparare.

La comprensione degli elementi fondanti della transizione in corso, da utilizzare nella re-invenzione dei propri modelli di business, è, tuttavia, ancora confusa. E, se guardiamo al dibattito corrente, spesso appare decisamente insufficiente, perché parziale o distorta.

Il digitale, infatti, non implica una trasformazione lineare, che agisca in modo univoco e uniforme nei vari settori e nei vari momenti della transizione. Al contrario, il digitale ha al suo interno una intrinseca polivalenza, perché cambia i modi di generare valore lungo tre traiettorie profondamente diverse, che sono sì complementari, ma anche - in parte contradditorie tra loro:

1) aumentando i moltiplicatori applicati al ri-uso di oggetti standard (dati, programmi, dispositivi, relazioni, significati ecc.), che possono essere replicati e propagati a costo zero (se immateriali e perfettamente codificati) o comunque a basso costo (se incorporati in un contenitore materiale, come una macchina, un prodotto, un'insegna pubblicitaria);

2) aumentando la varietà, la variabilità e l'interdipendenza della produzione on demand, che è in grado di generare valore addizionale fornendo una prestazione flessibile e personalizzata. Tale presentazione che, grazie al digitale, può oggi essere realizzata a costo contenuto, grazie ad automatismi digitali che sono in grado di gestire autonomamente la varianza codificabile (complicazione), usando algoritmi di apprendimento, modelli predittivi, sensori e "assistenti". Tutte cose che consentono ai dispositivi digitali di auto-situarsi e comunicare in modo interattivo col contesto;

3) aumentando la complessità, ossia la varianza non codificata che può essere esplorata dall'intelligenza fluida degli uomini, grazie anche all'impiego del digitale per ridurre i costi di esplorazione e sperimentazione delle possibili varianti utili. La crescita della complessità derivante dalla digitalizzazione apre spazi di libertà e ideazione creativa che in precedenza erano preclusi (per i loro costi e rischi eccessivi), dilatando la sfera del possibile e aprendo nuovi campi di generazione del valore. Diventa oggi possibile coltivare nuovi desideri e rispondere a bisogni caratterizzati da alta complessità ed empatia: la cura delle persone; la creazione di senso (attraverso lo sviluppo condiviso di nuovi legami e di nuovi significati); l'impiego ludico del tempo; l'impegno nella creatività artistica e artigianale; il turismo intelligente e la rigenerazione del paesaggio; le nuove concezioni di salute e benessere; lo sviluppo di responsabilità condivise nel rendere sostenibili le iniziative intraprese sul terreno sociale e ambientale.
Enzo Rullani

Nuovi modelli di business nel capitalismo globale della conoscenza in rete 


\section{sinergie}

Vol. 37, Issue 1, 2019

\section{Alla ricerca di nuovi modelli di business per il mondo digitale/ globale}

I modelli di business esistenti cambiano forma e traiettoria di sviluppo nel momento in cui si cerca di adeguarsi alle esigenze di una di queste tre forme di generazione del valore. Si tratta infatti di modificare le proprie valutazioni e proposte in uno dei tre modi prima richiamati: o moltiplicando gli standard; o personalizzando le varietà offerte; o esplorando la complessità libera, alla ricerca del nuovo. Oppure, combinando tra loro, in qualche modo, queste tre possibilità. Su questo terreno emergono, tuttavia, grandi differenze di approccio. In molte visioni della transizione in corso, il digitale viene visto come un medium che fa leva sulla prima di queste tre possibilità, moltiplicando le quantità del riuso degli standard e generando, così, un surplus di scala proporzionale al numero delle replicazioni. In questa ottica, si insiste soprattutto su valutazioni quantitative che misurano le replicazioni di un dato, di un video o di un programma fornito al mercato. Diventano così rilevanti il numero dei visitatori su un sito web, le folle più o meno estese di followers che seguono un influencer, le quantità di like ricevuti a commento di un tweet o di una foto, la massa dei dati impiegata per alimentare quantità di algoritmi predittivi ecc.). Il paradigma emergente finisce così per apparire la continuazione - con altri mezzi - del fordismo, riproponendo la sua logica di standardizzazione e di grandi volumi. Di conseguenza, i modelli di business pre-esistenti dovrebbero semplicemente dilatare la propria scala, con poche varianti sugli elementi di fondo: rimarrebbero valide, anche nella transizione attuale, la prevalenza delle grandi e grandissime dimensioni, lappiattimento sugli standard "vincenti", la replicazione pubblicitaria di massa di significati standard sempre più banali e semplificati.

Un passo avanti decisivo viene fatto quando, accanto alla replicazione degli standard si comincia a prendere in considerazione il secondo modo di generare valore: quello collegato alla crescita della varietà fornita al cliente. La capacità di robot, algoritmi e learning machines di gestire la varietà codificata $o$ facilmente codificabile (con forme automatiche di auto-apprendimento), rende ormai possibile aumentare la flessibilità e la personalizzazione dei prodotti/servizi offerti, variando anche con una certa facilità il disegno delle filiere produttive a valle e a monte, con l'impiego di una rete multipla di operatori interdipendenti, che lavorano on demand. In tal modo, si va decisamente oltre il fordismo, abilitando le iniziative decentrate di produttori (che offrono) e consumatori (che domandano), grazie a circuiti di relazione vocati alla flessibilità e alla personalizzazione.

Con l'impiego del digitale nella moltiplicazione degli standard e nella gestione automatica della varianza codificabile - le prime due modalità di generazione del valore - decolla una traiettoria evolutiva che enfatizza l'aumento della potenza tecnica della macchina, marginalizzando il ruolo dellintelligenza creativa degli uomini. L'uso intensivo di standard e automatismi digitali può, in effetti, creare grandi fatturati e - per quanto 
abbiamo detto ${ }^{1}$ - grandi surplus. Ma, nel corso del tempo, deve fronteggiare una inevitabile tendenza alla svalorizzazione dei prezzi realizzabili sul mercato. La concorrenza imitativa (facilitata dalla codificazione dei processi digitalizzati) alimenta infatti la tendenza, nel medio-lungo periodo, ad allineare il prezzo di mercato al costo di riproduzione del prodotto. Che, nel caso di prodotti ad alta intensità digitale, è zero o si avvicina allo zero. In altri termini, il tipico ciclo del valore di una innovazione di successo prima si impenna in modo esponenziale, quando gli standard e le personalizzazioni proposte sono nuovi e apprezzati dal grande mercato digitale/globale; ma poi - a meno di ricorrenti e costosi rinnovi del prodotto - i margini tendono a scendere, man mano che il prodotto/servizio offerto si banalizza, diventando scontato per i potenziali clienti.

Se le uniche direzioni di marcia della digitalizzazione fossero la moltiplicazione degli standard e la gestione automatica della complicazione, il risultato sarebbe alla lunga disastroso: labbattimento dei costi connesso alla digitalizzazione, a parità di domanda, finirebbe per espellere dal mercato una quota crescente di imprese e di lavoratori. Certo, la domanda di standard e di personalizzazione a basso costo potrebbe crescere, riassorbendo parte delle imprese e dei lavoratori espulsi. Ma il processo di banalizzazione conseguente alla caduta dei prezzi (e della scarsità corrispondente) rende improbabile una crescita compensativa adeguata. Dunque le visioni della transizione che si limitano a questi due effetti portano tutte, più o meno consapevolmente, all'idea di una transizione regressiva, dominata dalla disoccupazione tecnologica e da poteri monopolistici sempre più forti.

Le conclusioni invece cambiano se si considera il terzo effetto della digitalizzazione: l'aumento, progressivo ma inarrestabile, della complessità. Che deriva da due ragioni di fondo:

- gli standard e gli automatismi a basso costo rendono più facile, per gli uomini (imprenditori, lavoratori, consumatori, cittadini, comunità di senso, istituzioni) esplorare il nuovo, sperimentando il possibile senza eccessivi costi e rischi;

- il surplus che standard e automatismi mettono a disposizione dei produttori e degli users (con diverse ripartizioni a seconda della distribuzione del reddito co-prodotto nelle filiere digitali/globali) può oggi essere impiegato, in gran parte, per finanziare l'iniziativa intraprendente delle persone nellesplorazione della complessità, applicandosi a campi finora trascurati (De Toni e Rullani 2018). È questo il volano che, nella transizione in corso, permette di riassorbire

1 Se il costo di riproduzione delle conoscenze codificate è tendenzialmente zero - sia nel caso della moltiplicazione degli standard che in quello della complicazione affidata ad automatismi auto-referenti - il processo di replicazione della stessa base di conoscenza sarà in grado di generare un surplus proporzionale al numero dei ri-usi, visto che i costi sono per la maggior parte fissi, dando luogo ad un costo marginale basso o nullo, nella maggior parte dei casi. La rapidità della propagazione in rete degli standard o delle produzioni personalizzate determina così una corrispondente crescita esponenziale del fatturato e del surplus in valore, generato dalle filiere di co-produzione digitale/ globale.
Enzo Rullani

Nuovi modelli di business nel capitalismo globale della conoscenza in rete 
sinergie

Vol. 37, Issue 1, 2019 nelle dinamiche di lungo periodo le risorse di lavoro e di capitale che vengono rese disponibili dalla maggiore efficienza raggiunta attraverso la digitalizzazione degli standard e della varianza codificata ${ }^{2}$.

\section{Ri-posizionarsi, facendo surfing sulle cinque onde della transizione in corso}

Le tre modalità di creare valore attraverso il digitale, considerate sopra, devono tradursi in cambiamenti corrispondenti nei modelli di business delle imprese. Dando luogo a combinazioni in cui moltiplicazione degli standard, automazione della complicazione e esplorazione della complessità svolgono funzioni complementari, reciprocamente utili. E gestendo - aspetto non secondario, da considerare - le contraddizioni che emergono quando si cerca di integrare nella strategia, nell'organizzazione e nella pratica della stessa impresa principi per loro natura orientati in direzioni divergenti.

È questo il nuovo terreno di sfida per le innovazioni da introdurre nel modello di business delle imprese che vogliono sfruttare le potenzialità del paradigma emergente. Si può pensare che in questo ci sia qualcosa di déjà vu: da Schumpeter in poi sappiamo che il capitalismo si trasforma e si sostiene grazie al valore creato da innovazioni che operano qualche forma di "distruzione creatrice". Ma oggi - e qui sta la novità - innovare non basta più.

Perché, nel contesto della transizione, non tutte le innovazioni sono uguali: alcune usano infatti la forza delle grandi onde che, nel percorso delle digitalizzazione e globalizzazione, stanno cambiando il mondo; altre sono invece innovazioni di "ordinaria amministrazione", che forniscono prodotti e servizi utili ai clienti, ma senza avere particolari sinergie con le potenzialità tecnologiche del digitale e con le potenzialità moltiplicative del globale. Solo le prime riescono a "catturare" l'energia delle onde che, mentre l'azienda innovatrice va avanti, modificano in senso convergente la domanda dei clienti, le capacità dei fornitori, la potenza comunicativa

2 Va avanti, in questo modo, una traiettoria di evoluzione che prende avvio con la modernizzazione del mondo produttivo e di consumo di fine ' 700 . Prima sono state le macchine agricole a "spiazzare" il lavoro dei contadini nei campi, creando una forte disoccupazione tecnologica nel settore "primario", riassorbita in seguito - e solo lentamente - con lo sviluppo del settore "secondario" (la manifattura). Quando le tecniche fordiste che si affermano nel '900 riducono drasticamente al domanda di lavoro nelle fabbriche del "secondario", il gap occupazionale viene evitato perché il surplus in mano dei consumatori si traduce in una domanda crescente di servizi, alimentando la crescita compensativa del settore "terziario". Adesso, nel momento in cui standard digitali e complicazione automatizzata riducono l'impiego del lavoro contemporaneamente nel "primario", nel "secondario" e nel "terziario", la compensazione - graduale e niente affatto indolore - può essere ottenuta solo spostando il surplus disponibile su un settore "quaternario" che non è stato ancora individuato con precisione. Per quanto detto sopra, possiamo considerare che il "quaternario" di cui abbiamo bisogno è la crescita di complessità che nella transizione caratterizzerà sempre più i settori preesistenti. 
e logistica delle reti disponibili, le professionalità del lavoro. Dunque, bisogna scegliere in quale direzione e in quale senso orientare la propria nel capitalismo globale della vis innovativa e la propria assunzione di rischio.

Per realizzare il "salto" produttivistico necessario a colmare il gap di cui soffrono molte aziende su questo terreno, occorre elaborare e proporre innovazioni che siano pensate per fare surfing sulle grandi onde della transizione in corso. Solo queste possono contare, in prospettiva, su moltiplicatori e valori destinati a crescere, man mano che la transizione procede, come effetto della trasformazione generale del sistema produttivo e sociale.

Le cinque onde che funzionano come potenti attrattori dei nuovi modelli di business, da mettere in campo, sono:

- il networking, che porta ad operare in un mondo in cui tutti sono connessi con tutti, dando luogo ad una economia delle interdipendenze e delle relazioni radicalmente più profonda ed estesa di quella che abbiamo conosciuto in passato;

- l'automazione, che usa algoritmi e learning machines per governare non solo processi standard e ripetitivi, ma anche situazioni di complicazione, in cui si tratta di gestire una elevata varietà, variabilità e interdipendenza, attraverso dati predittivi e programmi di autoapprendimento che riescono a codificare le relazioni in essere;

- la globalizzazione delle reti cognitive, delle catene di produzione e dei mercati di sbocco, che crea valore attraverso lo sfruttamento non solo dei grandi moltiplicatori quantitativi, ma anche delle grandi differenze di costo e di capacità che esistono tra i paesi e i luoghi coinvolti;

- il worldmaking, ossia la creazione di "mondi" materiali, simbolici e identitari in cui vivere. Ė una tendenza che conferisce valore ai significati condivisi che i consumatori - ma anche i lavoratori, i finanziatori, gli imprenditori - assegnano alle attività svolte e ai servizi utilizzati. Grazie ai quali essi possono dare forma ad un mondo abitabile, conforme alle loro aspettative e alla loro sensibilità (essendo disposti, in moltissimi casi, a pagare un equo compenso per questo);

- la ri-personalizzazione, che mette nuovamente la persona al centro della scena produttiva, in tutti i casi in cui gli uomini sono chiamati a fornire l'intelligenza fluida necessaria per gestire la crescita esponenziale della complessità del mondo digitale/globale in formazione. La complessità è un problema sempre più rilevante - sia nella produzione che nel consumo - perché cresce l'incertezza e l'indeterminazione degli effetti futuri causati dalle azioni e dalle comunicazioni del presente. In questo contesto "liquido" ma aperto, le imprese e le persone hanno accesso ad ampie possibilità di esplorazione libera, che richiedono il giudizio, l'immaginazione, lassunzione di rischi da parte delle persone coinvolte.

\section{Ri-combinare standard, varietà e complessità nei modelli di business che cercano di sfruttare l'energia delle onde}

La sfida che porta ad integrare i tre driver della digitalizzazione (moltiplicazione degli standard, gestione automatica della complicazione, esplorazione human driven della complessità) nella gestione delle cinque 
Sinergie Vol. 37, Issue 1, 2019

grandi onde della transizione sopra ricordate vale per tutte imprese, grandi e piccole, e per tutti i paesi, avanzati ed emergenti. Ciascuno potrà e dovrà rispondere alle sfide a suo modo, sfruttando le pre-esistenze e trovando soluzioni ad hoc per superare gli ostacoli che si presentano sul cammino. In Italia, in particolare, le nostre imprese hanno discrete capacità di gestire il networking con altri attori rilevanti della filiera e della rete cognitiva utilizzata, ma devono ancora, in molti casi, imparare a:

a) codificare una parte rilevante delle conoscenze impiegate nella produzione e nella vendita, per renderle trasferibili in rete alle imprese e agli operatori che lavorano nella co-produzione del prodotto-servizio offerto, nella vendita rivolta al mercato e nelle attività di consumo;

b) creare e utilizzare reti efficienti di comunicazione delle informazioni e di trasferimento logistico delle merci (materiali), connettendo luoghi separati dalla barriera della distanza geografica, in forme assai diverse da quelle possibili in condizioni di prossimità fisica;

c) costruire relazioni trans-territoriali di fiducia e di condivisione che consentano di gestire in modo convergente le filiere e le relazioni col consumatore.

Per effetto del networking, l'uso degli standard e degli automatismi, ma anche l'esplorazione della complessità, possono acquistare forza attraverso ladozione dell'open innovation: i modelli di business che abbiamo ereditato dal secolo scorso sono ancora legati a confini ristretti, perché il fordismo (1900-1970) chiudeva le innovazioni ammesse nella gerarchia di potere organizzativo, mentre il capitalismo distrettuale (1970-2000) le chiudeva nellecologia del territorio.

Adesso, passando ad una logica di open innovation, diventa necessario invece aprirsi ad una logica di collaborazione con lesterno, cercando conoscenze, servizi, prestazioni utili nel grande mercato mondiale e selezionando i canali più promettenti. D’altra parte, è anche necessario che i significati associati ai propri prodotti o servizi possano arrivare su un mercato ampio, tendenzialmente globale.

Lapertura necessaria, a monte e a valle, mediata dal digitale, cambia in profondità il modello di business adottabile dalle imprese. Saltano molti dei precedenti confini tra luoghi e settori per cui emerge uneconomia in cui tutti possono essere, potenzialmente, chiamati a mettere le proprie capacità al servizio di tutti, nella logica della servitization. In parallelo, emerge il potere delle piattaforme: un potere ambivalente, che, da una parte, abilita gli users (imprenditori, lavoratori, consumatori, finanziatori, cittadini), potenziando le loro capacità decentrate di conoscenza e di condivisione; ma, dallaltra, concentra il potere di mercato e di gestione dei dati nelle mani di chi organizza la rete delle relazioni.

Cambia l'intermediazione: nella produzione on demand, i circuiti chiusi non reggono, perché ciascun anello della catena deve essere in grado continuamente di re-inventarsi e di ridefinire le relazioni con gli altri. E così via.

Si tratta di passaggi che sono particolarmente difficili per chi finora ha gestito con successo situazioni di interdipendenza locale, basate su relazioni interpersonali dirette, come è accaduto nella maggior parte dei distretti industriali italiani. Da un lato, servono investimenti e 
competenze che si possono mettere in campo solo progressivamente, nel corso del tempo; dall'altro, devono essere impiegate a questo scopo grandi piattaforme come Amazon, Alibaba, Google, Facebook, Booking ecc., se si vuole entrare in relazione con fornitori, clienti e "mondi" rimasti sinora lontani. Ovviamente pagando un prezzo - qualche volta non banale - alla mediazione di networking acquistata dalle piattaforme prescelte.

In questa proiezione verso l'open innovation, le imprese italiane maggiormente dinamiche possono contare sul vantaggio che deriva loro dall'aver fatto già in passato esperienza di livelli importanti di outsourcing, imparando a gestire la dipendenza affidabile da altri operatori della filiera. A cui sono stati delegati investimenti, competenze, funzioni produttive utili al completamento del proprio processo di generazione del valore. Certo, mettere oggi in piedi una rete di interconnessione e interdipendenze, estesa a scala trans-nazionale e su base codificata, non è la stessa cosa di quanto è riusciti a fare, con un certo successo, in passato. Ma nelle piccole e medie imprese dei distretti italiani non ci sono le chiusure auto-referenziali che la storia passata ha iscritto nel DNA di molte grandi imprese, italiane ed estere, abituate a gestire l'autosufficienza su tutti i problemi strategici, prima che l'interdipendenza con altri.

Per quanto riguarda l'automazione, invece, le pre-esistenze in Italia sono soprattutto concentrate sul versante dell'offerta di macchine flessibili e, oggi, intelligenti (la meccatronica, il packaging, le linee di lavorazione avanzate sperimentate nell'automotive e in altri settori). Meno rilevante è l'esperienza fatta, in questo campo, dal lato degli users. La ragione è semplice: in passato la varietà e la flessibilità di un modello di produzione neo-artigianale (prodotti di nicchia, prototipi, soluzioni flessibili su misura del cliente) sono state ottenute rendendo informale la catena produttiva e demandando alle persone in essa impiegate lavorazioni poco codificate, realizzate senza schemi rigidi e precostituiti. Una sorta di lean production all'italiana che sfruttava le relazioni interpersonali di prossimità e il "capitale sociale" delle conoscenze sedimentate nel luogo. Oggi questo modello è sostenibile - ancora per qualche tempo - solo se la personalizzazione dei prodotti e servizi forniti al cliente, con lavorazioni on demand o quasi, avviene per prodotti di alta qualità, associati a prezzi tanto elevati da coprire i costi di lavorazioni ancora di tipo artigianale. $\mathrm{Ma}$ ormai le produzioni automatizzate si stanno estendendo dalla fascia bassa dei prodotti standardizzati a quella intermedia dei prodotti customizzati, in cui al marchio di qualità gli algoritmi digitali associano una forma, una misura o una iscrizione personalizzata, diversa da cliente a cliente, o segmentata per gruppi di potenziali custormers. Nell'abbigliamento, nelle calzature, nell'arredamento e in moltissimi altri prodotti del made in Italy, l'uso della produzione on demand attraverso automatismi si va, infatti, diffondendo anche nella fascia dei prodotti di qualità, costringendo le imprese italiane che presidiano molti di questi campi ad aggiornare i loro precedenti modelli di business.

Della globalizzazione abbiamo già detto in precedenza: essa resta una sfida che le nostre imprese possono affrontare solo utilizzando la capacità estensiva e la duttilità delle filiere: alcune da estendere dal locale al globale; altre da "adottare" come nuovi contesti di co-produzione e appartenenza. Il
Enzo Rullani

Nuovi modelli di business nel capitalismo globale della conoscenza in rete 
sinergie Vol. 37, Issue 1, 2019

digitale può offrire spazi di crescita quantitativa alle produzioni di nicchia, che replicano i loro standard sul grande mercato mondiale, ma anche alla crescita della varietà e della complessità che usa la contaminazione $\mathrm{e}$ l'interazione con i nuovi mercati come mezzo di apprendimento.

Il worldmaking, come abbiamo detto, è uno dei driver più importanti per la transizione dei modelli di business delle imprese italiane, che sono già - nel loro campo - abituate a spostare laccento dalla fabbricazione di prodotti in funzione della loro utilità "materiale" alla generazione di valore che passa dalla creazione di significati, legami e identità collegati ai prodotti materiali, una volta che questi sono collocati in un contesto di uso che ne definisce il senso condiviso. Conferendo un valore che possiamo definire "immateriale" alle soluzioni fornite al consumatore finale.

È vero che il digitale dà accesso oggi a grandi potenzialità nello sviluppo di standard comunicativi (il marchio, la reputazione) che replicano lo stesso standard semantico milioni di volte, su tutto il mercato mondiale. Si sfrutta così un costo di pubblicità e di relazione molto inferiore al passato. Ma è anche vero che i messaggi veicolati da tale standardizzazione semantica cambiano quando si passa all'uso di automatismi che "vendono" al cliente non più lo standard, ma la varietà codificata, ancorata alla personalizzazione delle soluzioni proposte userfriendly. Prende, infatti, maggior peso il "servizio al cliente" e il co-design con le diverse categorie di utilizzatori potenziali. Se poi si comincia a lavorare sul fronte dellesplorazione della complessità, la leva fondamentale diventa il sense-making, la condivisione di senso con coloro - consumatori, fornitori, lavoratori, finanziatori, sindaci ecc. - che devono accompagnare l'impresa intraprendente nel viaggio di scoperta del nuovo.

Attraverso questo viaggio, il marketing emergente in tanti settori sta cambiando segno: nellarredamento, ad esempio, non si vende più un oggetto (un appartamento, un mobile, un elettrodomestico, un infisso, sistema di illuminazione, un bagno, un antifurto) al consumatore finale, ma gli si propone un "mondo" in cui tutti questi elementi formano un ambiente di vita o di lavoro che sia riconoscibile e apprezzato. Ossia che corrisponda ai desideri e alle aspettative dell'user.

È quanto sta già accadendo per la moda, che ormai non vende più oggetti definiti dalle diverse linee produttive (abiti, scarpe, gioielli, borse, occhiali ecc.), ciascuna delle quali in passato aveva un circuito specifico di comunicazione (marchi, pubblicità) e di vendita (negozi). Oggi, ormai, si vende uno "stile di vita", materializzato in un insieme coerente di oggetti, da associare ad un significato riconoscibile, e qualche volta identitario: ad esempio, un modo di vivere sportivo, creativo, ricco, spigliato, privo di pregiudizi. Oppure, forte o indipendente da tutti, a seconda dei casi e delle preferenze individuali e di gruppo. Abiti, scarpe, gioielli, borse e occhiali cominciano così ad essere contrassegnati dallo stesso marchio e venduti nello stesso negozio monomarca, in quanto associati allo stesso "stile di vita".

Da questo punto di vista, il made in Italy ha alcune pre-esistenze forti. Ma sono in gran parte legati ad oggetti distinti in base ai processi manifatturieri da cui discendono. Oggi devono invece declinare il loro significato puntando al worldmaking, che supera i confini settoriali e ibrida 
i diversi oggetti (cosa che si può fare anche con le licenze, ma non è sempre facile). Il made in Italy deve inoltre superare un handicap importante, su cui cè ancora poca consapevolezza: la focalizzazione ossessiva sull'unicità esclusiva dei valori e significati associati all'italianità, ossia al marchio di origine. Una qualificazione certamente utile per vendere, ma che può essere usata anche per portare sul mercato prodotti di scarsa qualità, sfruttando la denominazione di origine come fonte di "rendita": uno strumento che fornisce vantaggi di marketing senza sopportare costi e assumere impegni coerenti col marchio adottato (Rullani, 2016).

La smaterializzazione del valore, che fa dipendere una parte rilevante del valore fornito al cliente dal significato simbolico o fiduciario fornito al cliente, è un passaggio non banale per un sistema che finora ha mantenuto un forte legame con la materialità del prodotto, facendo leva sulla lavorazione artigiana, sul rapporto diretto con i clienti, sulla tipicità definita dalla tradizione del territorio o sulle aspettative espresse da segmenti di consumatori finali "fedeli" da tempo ad un certo marchio o ad una certa tradizione. Si tratta di ridefinire in senso digitale/globale queste forme di creazione di valore attraverso i significati e la loro condivisione sociale, mettendo in conto non solo gli investimenti richiesti da questo obiettivo, ma anche i suoi ritorni (maggiori moltiplicatori quantitativi, maggiore qualità anche in termini di prezzo).

Già nella creazione di significati condivisi che generano valore, emerge la forza della quinta onda della transizione: la ri-personalizzazione del mondo della vita e della produzione. Finora la modernità è andata avanti mettendo al centro di tutto la potenza produttiva delle macchine, intese come mezzo efficiente per ottenere un fine (la soddisfazione al costo minimo possibile di un bisogno materiale). Ma ciò è avvenuto, fino all'avvento del digitale, con l'impiego di macchine rigide, capaci di lavorare in modo replicativo e standard. Cosa che ha imposto una drastica compressione della complessità ammessa: nella fabbrica, nelle relazioni e nel consumo la varietà è stata ridotta a standard, la variabilità è stata ingessata in piani e procedure predeterminate, l'interdipendenza è stata ridotta al minimo (massima integrazione verticale delle grandi imprese fordiste, piramidi organizzative verticali al servizio del comando topdown). L'indeterminazione è stata attivamente combattuta, con tutti i mezzi possibili, organizzando sistemi stringenti di programmazione e controllo.

La presenza di macchine rigide in fabbrica e di beni standard (ottenute da esse) nel consumo ha finito per imporre le esigenze standard della macchina alle persone: il lavoro è diventato impersonale (tempo-lavoro); il consumo si è adattato al prodotto di massa; la fabbrica, ma anche tutta vita urbana e sociale intorno ad essa, è stata plasmata dalle esigenze efficientistiche delle macchine, assumendo di conseguenza forme e regolazioni standard, indifferenti alle diversità e aspirazioni delle persone.

Insomma, si è verificato un tipico caso di eterogenesi dei fini: quello che era un mezzo (la macchina), è diventato un fine auto-referente (Rullani e Rullani 2018). Rendendo impersonale il mondo della produzione, del consumo e delle relazioni sociali. Oggi siamo alla vigilia di grandi cambiamenti su questo versante, perché il digitale per un verso aumenta
Enzo Rullani

Nuovi modelli di business nel capitalismo globale della conoscenza in rete 
sinergie Vol. 37, Issue 1, 2019

la complessità del mondo di vita e di lavoro (per la maggiore varietà, variabilità, interdipendenza, indeterminazione già viste) e per un altro ci dà i mezzi per rendere governabile questa maggiore complessità. Ammettendo spazi di esplorazione creativa del nuovo e di creazione di senso condiviso, a cui attribuire valore.

È una trasformazione del mondo in cui l'Italia ha qualcosa da dire, oltre che molto ancora da imparare. Perché forse il nostro è uno dei sistemi in cui - grazie al "ritardato" e parziale avvento del fordismo nelle strutture produttive e sociali - le persone hanno conservato uno spazio di iniziativa e di differenziazione importante. Oggi si tratta di potenziare questo "capitale sociale" che deriva dalla tradizione investendo sulle capacità delle persone di ricollocarsi nella transizione digitale/globale sia in termini di istruzione che di condivisione sociale: due campi in cui il ritardo è documentato dalla bassa presenza di laureati e dallelevato individualismo degli imprenditori "personali" che, pure, hanno dimostrato di avere ottime capacità di iniziativa. Molte cose restano dunque da fare, anche se non siamo allanno zero.

\section{Problemi aperti, e innovazioni collettive necessarie per affrontarli}

Nella re-invenzione dei modelli di business delle imprese, accanto alle potenzialità, emerge, sotto molti aspetti, quello che è stato chiamato il dark side della transizione: un insieme di problemi irrisolti e spesso nascosti, che vanno oltre il campo di azione della singola impresa. E che richiedono dunque, per essere affrontati con successo, innovazioni collettive progettate e realizzate da soggetti sociali e politici capaci di affiancare le imprese e le singole persone. Questi soggetti, oggi, esistono ma non sempre appaiono consapevoli dei cambiamenti che, per quanto detto, dovrebbero organizzare e regolare. Se si vuole raggiungere qualche risultato apprezzabile su questo versante, è necessario che le associazioni imprenditoriali e i sindacati dei lavoratori; le istituzioni finanziarie; le organizzazioni dei consumatori; le istituzioni locali dei territori e gli Stati nazionali, con le loro (per adesso imperfette) proiezioni trans-nazionali partecipino attivamente al processo di de-costruzione del vecchio paradigma e di costruzione del nuovo.

Si tratta infatti di affrontare temi che mettono insieme una responsabilità di impresa con responsabilità sociali e politiche convergenti per sanare gli effetti di rottura, che stanno maturando con l'espansione spontanea, non regolata, della rivoluzione digitale/globale in atto. In alcuni campi critici:

a) la svalorizzazione del lavoro esecutivo;

b) l'aumento del rischio diffuso;

c) l'insostenibilità generata dal controllo di automatismi non-responsabili;

d) la crescita delle disuguaglianze sociali.

Come abbiamo visto, col procedere della digitalizzazione, il lavoro esecutivo che finora ha operato nella moltiplicazione degli standard o nell'automazione della varietà, scompare o si svalorizza, perché le funzioni di mera esecuzione di compiti codificati da procedure, calcoli o programmi vengono sempre di più affidate ad automatismi digitali a basso costo o decentrate in paesi emergenti (ugualmente a basso costo). Per prevenire 
e correggere gli effetti dirompenti di questa tendenza (disoccupazione di massa o comunque emarginazione di fasce rilevanti di lavoro in luoghi e funzioni "povere") è necessario fin da ora riconfigurare il lavoro e il rapporto fra impresa e lavoro, in modo da trasformare molti degli ruoli esecutivi in attività che invece richiedono intelligenza e intraprendenza creativa da parte del lavoratore. Specie se questi è incaricato di presidiare problemi complessi, che le macchine non possono svolgere da sole e che non possono nemmeno essere decentrati nei paesi low cost.

Le forme di smartworking che vanno oltre il lavoro esecutivo devono, su questo versante, sposarsi a politiche di impresa e del territorio mirate ad innalzare la qualità dei processi, attraverso innovazioni e investimenti adeguati nel digitale e nel globale. Non solo per consentire al lavoratore smart di sperimentare le sue nuove capacità su problemi e processi innovativi, in linea con i tempi; ma anche per creare una cornice di coinvestimento sul nuovo che metta insieme l'investimento di tempo e di attenzione fatto dal lavoratore con quello, convergente, fatto dall'impresa.

La crescita della complessità che si accompagna alla digitalizzazione, e che viene alimentata dallo spostamento del surplus su temi e significati complessi, rende - sulla carta - praticabile questo radicale cambiamento dei compiti e delle responsabilità assegnati al lavoro. Ma tutto dipende dalla rapidità con cui ogni paese e ogni gruppo sociale si muove in questa direzione. Non è infatti detto che la nascita dei nuovi posti di lavoro (intelligente e intraprendente) avvenga in luoghi, settori e tempi che coincidono con la perdita dei posti di lavoro esecutivo, sostituito da macchine o da lavoro low cost nei paesi emergenti. Si tratta di mettere in conto dei gap di luogo, di settore e di tempo che devono essere prevenuti o corretti, pena lo sviluppo di resistenze crescenti alla transizione verso il nuovo paradigma.

Il secondo problema di difficile gestione è l'aumento del rischio, connesso con l'instabilità creata dalle interdipendenze in tempo reale che caratterizzano la rete digitale. Abbiamo visto le molte ragioni per cui - con i nuovi cicli esponenziali del valore (in crescita e in decrescita) - il sistema che sta prendendo forma è intrinsecamente instabile. Il futuro atteso è quasi sempre un mix di promesse e di minacce difficilmente decifrabile. Dunque, il rischio crescente è diventata una condizione generale, che caratterizza tutti gli attori in gioco nella transizione.

Inoltre, bisogna considerare il fatto che non ci sono più quei "catalizzatori" del rischio (le maggiori imprese, le relazioni corporative, lo Stato regolatore e correttore) che in passato, con il loro potere di programmazione e concertazione, di fatto esentavano dal rischio i dipendenti (posto di lavoro "sicuro"), i risparmiatori (dividendo stabile), i consumatori ("fedeltà" alle promesse del marchio), i diversi attori della catena (forniture programmate o, comunque, aspettative affidabili di acquisto o di vendita), gli abitanti di un territorio, i cittadini dello Stato ecc.

In passato, l'assunzione di rischio da parte dei soggetti regolatori dei mercati e delle istituzioni aveva una contropartita importante: la concentrazione del rischio a carico di alcuni soggetti "forti" era, infatti, la premessa di una corrispondente concentrazione del potere decisionale. La valutazione dell'incertezza e le scelte da fare in merito, nella value chain, 
sinergie Vol. 37, Issue 1, 2019

erano infatti delegate a chi, in cambio, assumeva di fatto rischio delle conseguenze attese, esentando le controparti contrattuali.

Oggi, non solo il rischio è cresciuto insieme alla complessità, ma non ci sono più soggetti abbastanza "forti" e consolidati da essere disposti ad assorbirlo, esentando gli altri. Le grandi imprese cercano infatti di avere, al massimo, le "mani libere" per potersi riposizionare in funzione di cambiamenti emergenti, che - essendo in buona parte fuori del loro controllo - dunque, risultano poco o per niente programmabili. Lo stesso vale per gli Stati: la finanza pubblica ha ormai margini esigui di intervento per assorbire rischi diffusi e "pesanti", gravanti sulla popolazione.

La conseguenza è che le imprese, i lavoratori, i risparmiatori, i cittadini devono imparare a convivere con un rischio diffuso, che grava sulle loro spalle. E che - a parte lo stato di ansia che ne deriva - ostacola gli investimenti sul futuro richiesti a ciascuno di essi per acquisire le capacità necessarie ad operare nel nuovo paradigma digitale/globale.

Ne sono un sintomo visibile le resistenze con cui le imprese oggi valutano la possibile assunzione di un neo-dipendente utilizzando il frame contrattuale che, nel fordismo, era la norma: quello del lavoro subordinato a tempo indeterminato. Un contratto del genere, considerate le difficoltà che la regolazione contrattuale e pubblica ha introdotto, nel corso del tempo, a tutela del lavoratore, costituisce - per l'impresa - una sorta di sunk cost da cui è difficile recedere, in caso di necessità. E, dunque, si tratta di un contratto che, implicando un trasferimento del rischio dal dipendente allimpresa, sta diventando problematico. Le imprese sono, infatti, sempre di più riluttanti di fronte a scelte di assunzione che le immobilizzano su una certa posizione, in presenza di possibilità future non programmabili. Molte di esse preferiscono altre forme contrattuali (lavoro temporaneo, di somministrazione o addirittura autonomo con partita iva), meno vincolanti. O scelgono di decentrare funzioni e fasi della filiera a fornitori esterni, accettando così una logica di flessibilità che rende conveniente l'outsourcing da altre imprese (un impegno revocabile in ogni momento). Ma questo limita al minimo necessario gli investimenti da fare e la dimensione di impresa da conseguire nel corso del tempo: ciascuna impresa finisce per presidiare soltanto il core business distintivo, lasciando il resto agli altri.

Con una conseguenza esiziale: se tutti cercano di avere le mani libere, infatti, il risultato è che nessuno investe sulla creazione di conoscenze e strutture in un orizzonte di medio o lungo periodo. Il processo di adeguamento del sistema alle esigenze del paradigma digitale/globale emergente finisce per arretrare o bloccarsi del tutto, perché non si trova il modo per tenere insieme la flessibilità dell'impresa con un investimento rilevante da fare in human capabilities, in relazioni affidabili, in significati condivisi e impegnativi.

Anche in questo caso, il vecchio gioco del "cerino" (passare il cerino acceso ad altri, in modo che sia qualcun altro a scottarsi) finisce per generare solo conflitto e insicurezza reciproca, minando la condizione di base per la divisione del lavoro cognitivo e operativo: la reciproca affidabilità.

Dunque, la soluzione da mettere in cantiere è unaltra: il rischio che non si riesce ad eliminare, né a passare ad altri, va condiviso. In modo 
trasparente e consapevole, creando contratti di tipo nuovo nelle relazioni industriali (tra capitale e lavoro), nella filiera (tra fornitori e clienti), nel finanziamento (tra azienda e risparmiatori-investitori), nel territorio (tra azienda altri attori locali), nelle relazioni sociali (con i consumatori, le comunità di senso, le istituzioni). In tutti questi contratti, bisogna seguire una logica nuova, partendo dallo sviluppo di progetti di innovazione comuni, per arrivare a condividere gli investimenti e costi richiesti, gli impegni assunti e i risultati alla fine conseguiti (in profitto o in perdita). Siamo all'inizio di questo percorso, ma la direzione di marcia è tracciata: bisogna che le imprese e gli attori con cui esse lavorano ne prendano consapevolezza, in modo da elaborare un senso condiviso delle loro azioni.

Un altro problema critico, che non può essere affrontato a scala individuale, è l'emergere di situazioni di insostenibilità, determinate, in modo sempre più esteso, dallo sviluppo spontaneo delle innovazioni dirompenti che sfruttano il potenziale della transizione digitale. Quando le scelte di mercato sono lasciate ai singoli e sono orientate su convenienze di breve o brevissimo periodo, gli effetti di insostenibilità sono inevitabili, anche se i danni qualche volta emergono subito, altre volte più lentamente.

L'insostenibilità è una delle caratteristiche fondamentali dello sviluppo avviato dalla modernità, nei due secoli e mezzo trascorsi dalla rivoluzione industriale ad oggi. Non è dunque un problema nuovo: anche in passato, tutti gli automatismi della modernità sono sempre andati avanti in modo auto-referente, massimizzando l'efficienza riferita al proprio obiettivo funzionale (Rullani 2010). La tecnologia ha cercato innanzitutto di aumentare le potenza dei mezzi nel dominio della natura, il calcolo di convenienza ha badato soprattutto ad ottimizzare le scelte orientate al profitto di impresa; il mercato di concorrenza ha presidiato la selezione delle soluzioni e routines più efficienti; l'organizzazione, di matrice fordista, ha cercato fino all'ultimo di garantire la stabilità delle relazioni tra i poteri consolidati; le procedure organizzative e le norme istituzionali hanno assunto come proprio compito la disciplina di specifici comportamenti.

Tutti questi comportamenti, focalizzati sul fine particolare di questo o quellautomatismo, hanno nel tempo creato forti "diseconomie" e disfunzioni su aspetti della vita sociale e dell'ambiente naturale eccedenti la loro auto-referenza. È accaduto cento volte nella storia passata, alimentando l'inquinamento dell'ambiente, del clima organizzativo e della qualità della vita nel contesto sociale. Inizialmente ciò si è mantenuto entro margini di tolleranza accettabile; ma, alla fine, si sono create molte situazioni di vera e propria insostenibilità, bloccando lo sviluppo di tutti quei processi che - a causa della loro inconsapevolezza dissipativa - non riescono a riprodurre le loro premesse. È così che si sono create in molti campi le condizioni per la stagnazione e per un disagio diffuso, fortemente conflittuale. A cui, finora, si è cercato di rispondere utilizzando la politica e la responsabilità sociale. Con alterne fortune.

Le situazioni di insostenibilità generate dal dark side del digitale, si aggiungono, dunque, ad una problematica ben nota. Tuttavia oggi sta venendo in evidenza una novità importante: gli automatismi digitali tendono - per aumentare la loro efficienza nella replicazione degli standard e nella gestione della complicazione - a usare in modo poco trasparente
Enzo Rullani

Nuovi modelli di business nel capitalismo globale della conoscenza in rete 
sinergie Vol. 37, Issue 1, 2019

e autoritario i dati relativi alla vita e al lavoro delle persone coinvolte nei processi produttivi. E questo non solo per aumentare le capacità predittive degli algoritmi (ossia la loro potenza dispositiva), ma anche per condizionare attivamente le scelte delle persone, violando la loro privacy $\mathrm{e}$ la libera formazione delle loro opinioni. L'insostenibilità rischia in questo modo di diventare presto una caratteristica invasiva e distorsiva delle relazioni cognitive e sociali, oltre che economiche, emergenti dal nuovo paradigma.

Servono rimedi collettivi per correggere questa tendenza implicita della digitalizzazione. Rimedi che cominciano ad essere sperimentati, anche se in forma per adesso soltanto iniziale: norme sulla trasparenza dei dati, tutela della privacy, regolazione delle grandi piattaforme intese come beni comuni, disciplina fiscale adeguata alla nuova distribuzione del surplus che si realizza nelle filiere digitali/globali. Ma se si vuole recuperare agli uomini - e alla loro libera scelta - la gestione delle relazioni, sottraendola davvero allefficienza "cieca" o egoista degli automatismi digitali, serve altro: bisogna guardare ai nuovi spazi di responsabilità, di condivisione e di sense-making che si aprono, nellesperienza di ciascuno, per effetto del progressivo aumento della complessità del mondo della vita e del lavoro. Un universo di possibilità in cui la delega agli automatismi di alcune funzioni non potrà che essere affiancata dalla costruzione di comunità di senso, in cui le persone - con la loro intelligenza fluida e la loro empatia proveranno a immaginare e dare forma ad un mondo (per loro) abitabile, e (per loro) dotato di legami e di senso.

Infine, uno dei problemi più importanti di ingiustizia (ma anche di insostenibilità) che emergono nellesperienza attuale è quello della crescita delle disuguaglianze sociali. È sicuramente un problema meno netto e lineare di quanto appare nelle ricostruzioni che attribuiscono al "capitalismo digitale" una intrinseca finalità di espropriazione sociale. Ma certo è un problema fondamentale, cui occorre trovare rimedio per costruire un paradigma digitale/globale capace di esprimere al meglio le sue potenzialità di valore e di senso.

Intanto, nella rappresentazione del fenomeno bisogna distinguere tra le disuguaglianze tra paesi e quelle che sono invece interne ai singoli paesi.

La rapida crescita della Cina e di altri paesi emergenti promette in breve tempo di porre rimedio a disuguaglianze strutturali di reddito e di condizioni di vita tra paesi che erano state ereditate dal secolo scorso e che erano rimaste finora senza rimedi sostanziali. Il mondo oggi comincia ad essere meno diseguale di quanto era nel 2000 , grazie alla rapida crescita dei paesi emergenti e il loro ruolo, sempre più rilevante, nelle global value chains.

$\mathrm{Ma}$ è anche vero che le disuguaglianze sono cresciute allinterno dei paesi sviluppati. Trasformandosi in una frattura tra quelle che sono state chiamate le élites e il resto della popolazione (il "popolo"). È una frattura che oggi separa i grandi centri urbani città (aperti al globale e alle sue contaminazioni) dalla periferia (chiusa nella conservazione degli assetti e della cultura pre-esistente). Ma distingue anche tra la parte della popolazione maggiormente istruita, capace di operare senza difficoltà nelle reti digitali/globali di oggi, e la popolazione meno dotata di conoscenze e 
capacità utili a questo scopo, risultando così emarginata e resistente alle innovazioni emergenti. In certi paesi, come l'Italia, sta prendendo corpo anche una demarcazione tra lavoratori occupati e disoccupati, tra regioni del Centro-Nord e quelle del Sud, tra imprese intraprendenti e attori inerziali, ancorati a modelli del novecento.

Le linee di demarcazione della disuguaglianza sono tante, molte delle quali appaiono oggi "calde" e talvolta incandescenti. Ma il problema è di adottare - sui vari conflitti emergenti dalla disuguaglianza - un atteggiamento pragmatico che riesca a portare progressivamente a sintesi gli interessi divergenti delle parti in causa. Senza bloccare l'evoluzione verso il nuovo, che è necessaria per avere a disposizione il valore addizionale generato dalla transizione digitale. Questo valore è necessario non solo per sopravvivere alla concorrenza in atto, ma anche per affrontare i disagi creati dalle innovazioni più dirompenti, foriere di maggiore disuguaglianza.

Per gli interventi urgenti è senz’altro ragionevole una ridistribuzione del surplus ottenuto dalle innovazioni digitali/globali introdotte. Ma guardando al lungo termine, è meglio non focalizzare l'azione correttiva su obiettivi meramente assistenziali, che - una volta raggiunti - finirebbero per "congelare" e rendere permanenti le disuguaglianze attuali. Il percorso correttivo più coerente con le possibilità del paradigma digitale/globale è invece quello che mira a creare le condizioni che consentano di rimettere "in pista" coloro che, per adesso, sono rimasti esclusi o marginalizzati. E che devono essere aiutati a inserirsi in una prospettiva di investimento sulle capacità, di condivisione del senso e di collaborazione intraprendente. Tocca agli attori - individuali e collettivi - più sensibili alla responsabilità sociale e alle esigenze della sostenibilità rendere possibile questo processo di condivisione dei costi, dei significati e dei rischi tra i "vincenti" e i "perdenti" iniziali della trasformazione in corso.

Le imprese sono sicuramente tra gli attori maggiormente coinvolti dall'emergere dei problemi critici ricordati sin qui (svalorizzazione del lavoro esecutivo, rischio diffuso, insostenibilità, disuguaglianza). Lo sono oggettivamente, perché la loro ricerca di percorsi efficaci di transizione dal vecchio al nuovo rischia di essere intralciata, anche seriamente, dall'emergere di problemi che rendono precario l'ambiente in cui i nuovi modelli di business delle imprese dovrebbero prendere forma. $\mathrm{Ma}$ possono e devono esserlo anche soggettivamente, se diventano parti attive di comunità di senso in cui viene condiviso il significato e la responsabilità di processi di crescita partecipati e inclusivi.

Si tratta di un percorso lungo e accidentato, certamente. Ma l'importante è acquistare piena consapevolezza della posta in gioco e partire, iniziando a sperimentare, in modo responsabile e condiviso, le nuove possibilità che la transizione rende accessibili.

E avere la pazienza di andare avanti con determinazione. Anche un lungo cammino comincia sempre con un primo passo.
Enzo Rullani

Nuovi modelli di business nel capitalismo globale della conoscenza in rete 


\section{sinergie}

Vol. 37, Issue 1, 2019

\section{Bibliografia}

DE TONI A., RULLANI E. (2018) (a cura di), Uomini 4.0: ritorno al futuro. Creare valore esplorando la complessità, Cfmt e Un. di Udine, Franco Angeli, Milano.

FRIEDMAN T.L. (2005), The World is Flat, traduzione italiana Il mondo è piatto. Breve storia del ventunesimo secolo, Mondadori, Milano, 2006.

RULLANI E. (2010), Modernità sostenibile. Idee, filiere e servizi per uscire dalla crisi, Marsilio Editore, Venezia.

RULLANI E. (2016), "Il futuro del made in Italy: una sfida da condividere", Premessa al volume di Gregori G. L. (a cura di), Made in Italy. Una lettura critica tra eredi virtuosi e dissipatori, Il Mulino, Bologna, pp. 9-22.

RULLANI F., RULLANI E. (2018), Dentro la rivoluzione digitale. Per una nuova cultura dell'impresa e del management, Giappichelli, Torino.

\section{Academic or professional position and contacts}

\section{Enzo Rullani}

Full Professor of Management

TeDIS Center - Venice International University

e-mail: enzo.rullani@gmail.com

sinergie

ISSN 0393-5108 DOI $10.7433 / \mathrm{s} 108.2019 .02$
pp. $17-34$ 